\section{REVISTA BRASILEIRA DE QUALIDADE DE VIDA}

\title{
Os benefícios da musculação para a vida social e para o aumento da auto-estima na terceira idade
}

\section{The benefits of strength training for social life and increase self-esteem in old age}

\author{
Marco Antonio Bettine de Almeida \\ Escola de Artes, Ciências e Humanidades - Universidade de São Paulo - São Paulo - SP - Brasil \\ marcobettine@usp.br
}

Barbara Pavan Escola de Educação Física e Esporte de Ribeirão Preto - Universidade de São Paulo - São Paulo - SP - Brasil barbara.pavan@usp.br

\begin{abstract}
Resumo
O objetivo deste trabalho, por meio de revisão de literatura, identificar os benefícios que traz a musculação para a vida social e para a auto-estima em idosos acima de 60 anos. O método de análise baseou-se na análise bibliográfica e textual. O Resultado do estudo revela que a musculação não é a protagonista na melhora da vida social e da auto-estima, mas auxilia de maneira integrada com demais atividades físicas. A melhoria na vida do idoso acontece através de uma somatória de fatores físicos, subjetivos e sociais. Conclui-se que os benefícios na vida social e na auto-estima na terceira idade não é consequência apenas do exercício de força e sim da associação desta modalidade com outras atividades físicas, através da integração entre os participantes e melhoras na qualidade de vida.
\end{abstract}

Palavras-Chave: Terceira Idade, Musculação, Benefícios.

\section{Summary}

This study, through literature review, identifying the benefits that brings fitness for social life and self-esteem in individuals over 60 years. The analysis method was based on literature and textual analysis. It was possible to see that bodybuilding is not the protagonist in the improvement of social life and self-esteem, but assists in an integrated manner with other physical activities. The improvement in the lives of the elderly occurs through a summation of physical, social and subjective. It might be considered that the benefits in social life and self-esteem in old age is not only a consequence of the exercise of force, but the combination of this modality with other physical activities, through integration between the participants and improvements in quality of life. Keywords: Elderly, Bodybuilding, Benefits.

\section{Introdução}

Doenças geriátricas no Brasil começam aos 40 anos, idade em que surgem os grandes problemas degenerativos, coronariopatias, diabetes e hipertensão. Principalmente pelos maus hábitos alimentares, sedentarismo, stress e desnutrição. A velhice além de alterações biológicas traz 
mudanças psicológicas e sociais que contribuem para o pouco relacionamento do idoso consigo mesmo, com a família, amigos e a sociedade. Os exercícios físicos trazem muitos benefícios na terceira idade, conforme citações de Nadeau e Peronnet (1985). Eles aumentam a massa muscular, reduzem o percentual de gordura corporal, aumentando a força do indivíduo, facilitando a sua locomoção, mantêm a pressão sanguínea e a frequência cardíaca dentro de padrões aceitáveis para a idade, dificultando o acúmulo de colesterol no sangue.

A musculação faz com que o indivíduo tenha mais força, devido ao aumento da massa muscular evitando quedas, pois $40 \%$ das pessoas acima de 65 anos caem pelo menos uma vez por ano, podendo ocorrer lesões, principalmente as fraturas que reduzem a mobilidade articular.

É crescente hoje o número de pessoas preocupadas com a saúde dentre elas a terceira. $\mathrm{O}$ exercício físico com ênfase em força pode ser uma boa saída para que eles fortaleçam os seus músculos e vivam melhores e mais independentemente. Este artigo tem o intuito de aprofundar os conhecimentos na área da musculação dando ênfase aos possíveis benefícios que essa atividade pode trazer á vida social e a auto-estima de idosos, e de não ver a musculação apenas como um benefício á saúde física desse grupo.

Nahas (2001) escreve que benefícios da atividade física a partir da meia idade podem ser analisados na perspectiva individual ou social. Nahas coloca as vantagens da seguinte maneira: benefícios fisiológicos - controle dos níveis de glicose, maior capacidade aeróbia, melhoria na flexibilidade e equilíbrio, benefícios psicológicos - relaxamento, redução na ansiedade e melhoria na saúde e diminuição no risco de depressão e por fim benefícios sociais - indivíduos mais seguros, maior integração com a comunidade e funções sociais preservadas.

Diante disso a pratica de atividade física não é só importante para a força física, mas um bem necessário para fugir da depressão e conviver socialmente. $\mathrm{O}$ idoso é beneficiado como um todo ao realizar o exercício físico, pois numa sociedade que envelhece rapidamente é fundamental que se redefina o papel do idoso no âmbito social, valorizando assim a contribuição que ele ainda pode trazer.

\section{Aspectos Físicos do Envelhecimento}

Para que o processo de aumento da massa muscular ocorra com eficiência, Silveira Júnior (2001) observa que não basta oferecer o estímulo do treinamento físico, também é necessário manter o organismo em situação metabólica favorável. Esta situação é a predominância do anabolismo sobre o catabolismo, ou seja, das reações de síntese sobre as reações de degradação de matéria.

Alimentação e repouso (sono) são variáveis muito importantes nesse processo. O aumento da ingestão calórica, juntamente com a ingestão adequada de proteína (1,6 a 1,8 gramas por quilo de peso corporal) e ingestão reduzida de gorduras, sobretudo, as saturadas, é o princípio alimentar mais importante para ganhos de massa muscular durante o treinamento de força. A ingestão adequada e regular de carboidratos é um fator importante no treinamento de força, pois esse nutriente, além de ser o principal combustível energético para o sistema nervoso central e para o exercício, possui a função de ajudar na preservação das proteínas teciduais, evitando ou amenizando a gliconeogênese realizada pelo fígado para a transformação da proteína ou gordura em glicose, quando os níveis de glicose ou as reservas de glicogênio estiverem baixos. Em condições extremas, esse processo acarreta uma redução significativa da massa corporal magra e sobrecarga renal.

Podem-se destacar alguns tipos de adaptações básicas ao treinamento de força, as de ordem neural, metabólica e fisiológica e as de ordem morfológica. Dependendo da metodologia empregada (força, resistência de força ou potência), certas adaptações serão mais enfatizadas que outras, associando ênfase adaptativa à capacidade biomotora treinada.

Atualmente, tem-se atribuído grande importância ao treinamento de força para a manutenção da saúde, na população em geral. Tendo em vista essa importância, o entendimento dos mecanismos responsáveis pelas adaptações decorrentes do treinamento dessa capacidade motora torna-se primordial para a sua maximização. Pode-se afirmar que a prática permanente de atividade física 
melhora as condições gerais do ser humano e seus resultados são significativos com a intensidade adequada, respeitando a individualidade biológica, melhorando os benefícios a médio e longo prazo, obtendo-se um menor desgaste físico, mais segurança e saúde.

Não poderia passar despercebida uma melhora na mobilidade articular e, principalmente, na sociabilidade e questão emocional (mais vontade de viver), observada com mais intensidade no grupo de maior idade.

Há certo consenso sobre a influência positiva do exercício físico com sobrecarga, inclusive na terceira idade. Mulheres e homens experimentam importantes adaptações fisiológicas e de desempenho ao treinamento de resistência independentemente da idade. Um estudo com homens idosos e sadios (com media de 68 anos) demonstra a impressionante plasticidade do músculo esquelético humano. Os homens treinaram por 12 semanas utilizando exercícios com resistência pesada, isocinéticos e com pesos livres. O treinamento fazia aumentar o volume dos músculos e a área em corte transversal do bíceps braquial $(13,9 \%)$ e do braquial $(26,0 \%)$ enquanto a hipertrofia aumentava em $(37,2 \%)$ nas fibras musculares tipo II. Aumentos de $46 \%$ no torque máximo e de $28.6 \%$ na produção total de trabalho acompanhavam as adaptações celulares.

Os homens mais velhos experimentam aprimoramentos percentuais nessas variáveis semelhantes aos seus congêneres mais jovens, em resposta a um rápido programa de treinamento de resistência periodizado com alta potência. Respostas ao treinamento igualmente impressionantes ocorrem para as pessoas idosas.

Os residentes de asilo (com media de 87,1 anos) treinaram por 10 semanas com um exercício resistido de alta intensidade. Para as 63 mulheres e 37 homens que participaram a força muscular aumentou em media $113 \%$. Os aumentos de força mantinham também paralelismo com a velocidade da marcha $11,8 \%$ e velocidade para subir escadas 28,4\% (MATSUDO, 2005).

Algumas situações em que os exercícios com peso devem ter prioridades em relação aos aeróbicos: artrite severa, inabilidade para suportar o peso corporal, ulcerações no pé, desordem de equilíbrio, amputação, doença pulmonar obstrutiva crônica severa, alto risco de isquemia (MATSUDO, 2001).

Qualquer melhora, mesmo de maneira limitada, é extremamente significativa na qualidade de vida e na diminuição dos riscos de doenças relacionadas ao envelhecimento (CAMPOS, 2000).

De acordo com Matsudo (2001) e Okuma (2002) a capacidade funcional é considerada requisito primordial para a habilidade do idoso de viver em condições de independência podendo, desta forma, realizar tarefas simples e complexas do cotidiano sem necessitar a ajuda de ninguém.

A Classificação Internacional de Funcionalidade, Incapacidade e Saúde (CIF) define funcionalidade como um termo que abrange todas as funções do corpo, atividades e participação. As funções e estruturas do corpo são as funções fisiológicas dos sistemas orgânicos do corpo. Atividade significa a execução de tarefas ou ações pelo indivíduo, enquanto participação seria o envolvimento do indivíduo em situações do seu dia-a-dia. Em contrapartida, os aspectos negativos das estruturas e funções do corpo, atividades e participação, respectivamente denominados como deficiências, limitações da atividade e restrições da participação, recebem o nome de incapacidade.

Fried et al. (2004) define incapacidade como dificuldade ou dependência na realização de atividades essenciais para uma vida independente, incluindo atividades de autocuidado e aquelas consideradas importantes para a qualidade de vida do indivíduo. Incapacidade pode ser definida, em alguns contextos ampliados à saúde, como um fenômeno social. Jette (2003) relata em seu trabalho que, tradicionalmente, as medidas de incapacidade utilizadas em pesquisas gerontológicas são organizadas sobre o construto de atividades do dia-a-dia, das quais fazem parte as atividades básicas e instrumentais de vida diária.

As incapacidades físicas ocorrem com freqüência em idosos. Estima-se em torno de $20 \%$ a $30 \%$ o número dos indivíduos acima de 70 anos que vivem na comunidade e que relatam incapacidades para realizar tarefas que requeiram o uso da mobilidade e da deambulação, atividades de vida diária (AVD's) e atividades instrumentais de vida diária (AIVD’s). Essas incapacidades aumentam proporcionalmente com a idade, a partir de 65 anos (FRIED et al., 2004). 
Incapacidades físicas ao final da vida são resultantes das alterações fisiológicas do processo de envelhecimento, de doenças e problemas associados, cujo impacto pode ser modificado não só por fatores sociais, econômicos e comportamentais, como também pela possibilidade de acesso aos cuidados médicos (FRIED et al., 2004).

Doenças e a presença de co-morbidades, fraqueza muscular, alterações de equilíbrio ou diminuição da tolerância ao exercício e a fragilidade por si só são identificados como fatores de risco para a incapacidade física e podem agir de forma independente ou sinergicamente (FRIED et al., 2004).

Bons níveis de aptidão física em idosos têm se mostrado indicador importante sobre a melhoria de vários aspectos físicos e funcionais, por exemplo, bons parâmetros de composição corporal, controle de peso, capacidade funcional e condicionamento físico melhorado (MATSUDO, 2005; VALE; NOVAES; DANTAS., 2005; ZAGO; GOBBI, 2003).

Outro aspecto relacionado aos benefícios que a atividade física regular pode proporcionar aos idosos está na prevenção de quedas, pois conforme Dantas (2005) a atividade física auxilia em alguns elementos indispensáveis para que o idoso previna-se de eventuais quedas: fortalecimento dos músculos das pernas e costas; melhora dos reflexos, bem como a sinergia motora das reações posturais; velocidade de andar; incremento da flexibilidade; manutenção do peso corporal; melhora da mobilidade e diminuição do risco de doença cardiovascular. Guimarães e Farinatti (2005) revelaram, em um estudo realizado com mulheres idosas, os fatores que causam eventuais quedas em idosas, observou-se que a variável flexibilidade possuiu uma associação significante com as quedas.

Shephard (2003), afirma que são inúmeros os argumentos que encorajam os idosos a praticarem atividades físicas e manterem um estilo de vida ativo durante a velhice, pois além das questões relativas aos domínios físicos e funcionais, a prática de atividade física pode proporcionar aos idosos benefícios nos aspectos mais subjetivos como o social e o psicológico.

Erbolato (2002) afirma que na velhice é normal que exista uma diminuição dos contatos sociais, provocando estado de solidão nos idosos. Porém, o engajamento de indivíduos desta faixa etária em programas de atividades físicas poderá proporcionar a ampliação do círculo de amizades e, desta forma, contribuir no processo de integração social e elevando a auto-estima do idoso.

\section{Aspectos Sociais no Envelhecimento}

O processo de envelhecimento ocorre com todos os seres vivos, mas é com o ser humano que este processo acarreta desconforto, pois o tema velhice vem carregado de preconceitos. Para Monteiro (2001) e Okuma (1998), os atributos negativos ditados pela sociedade priorizam as características biológicas e físicas. Meirelles (2000) e Okuma (1998) completam dizendo que na nossa sociedade atual ser velho tem uma grande conotação negativa. O processo de envelhecer carrega um estereótipo social negativo muito grande, fundamentando uma idéia errada de que obrigatoriamente o envelhecimento causa "incompetência comportamental".

Nessa desvalorização o idoso diminui a sua participação no meio social, gerando um sentimento de inutilidade, levando-o a apresentar problemas orgânicos e psicológicos, ocasionando o isolamento social. Segundo Lorda e Sanchez (2001) na $3^{\text {a }}$ idade as muitas mudanças no ambiente social do idoso exigem dele uma constante adaptação. Existe por parte de alguns idosos a negação do seu próprio envelhecimento, provocando muitas atitudes, sendo que, algumas delas, prejudiciais a manutenção do seu lado psicológico, entre elas: o isolamento, levando-o a evitar contato com pessoas da mesma idade e fazer-se de vítima.

Além do receio da senilidade, as pessoas não aceitam o envelhecimento devido também a falta de uma "razão de ser" da velhice, por não encontrarem um papel para ele mesmo na sociedade. A própria sociedade, ao mesmo tempo em que o marginaliza por considerá-lo incapaz e improdutivo, impede que ele volte a ser capaz e produtivo. Isso ainda continua muito comum, mas existem alguns focos da sociedade que tem dado alguma chance para os idosos poderem mostrar 
sua verdadeira capacidade dependendo muito do próprio idoso se sentir bem e pronto para poder se reintegrar à sociedade.

A maioria dos idosos não consegue continuar desempenhando uma vida ativa, mesmo com uma saúde muito boa. Este é o primeiro impacto do envelhecimento para o indivíduo: a perda de seus papéis sociais e o vazio experimentado por não encontrar funções. Sente angústia, decepção e sofrimento. Geis (2003) destaca que a solidão é um outro desafio para os idosos. Com a aposentadoria, perde-se o grupo social do trabalho, a viuvez acontece e, geralmente, os filhos já estão casados, deixando uma imensa sensação de vazio.

O exercício físico é um instrumento de prevenção e manutenção da saúde psicossocial do idoso, pois, através de atividades prazerosas, as limitações próprias da idade se dissipam ou minimizam, colaborando para a integração social, desenvolvimento do espírito de equipe, restaura a independência e autonomia, promove a inclusão e maior habilidade frente às tensões, desinibe e desbloqueia, contribui para diminuir a ansiedade, a tristeza, a solidão e a impaciência.

Nos últimos anos houve um interesse científico representativo pela temática do envelhecimento, destacando a importância da prática regular de atividades físicas e uma intervenção nutricional adequada. Há uma tendência ao envelhecimento global e que a maioria dos efeitos degenerativos do envelhecimento é devido ao sedentarismo, imobilidade e desadaptação social.

Esse é o grande desafio para todas as áreas do conhecimento: atribuir qualidade de vida a essa população, que não só tem possibilidades de viver mais, porém merece viver com qualidade, dignidade e, principalmente, estimulando a auto-estima para a superação de pequenos desafios (OKUMA, 2002).

O termo "qualidade de vida" engloba diversos aspectos da vida de uma pessoa, tendo em vista, a população em geral. Do ponto de vista emocional, essa expressão é feita a uma pessoa que tem um grau de satisfação elevado com a vida. Segundo Nahas (2001), a qualidade de vida pode ser desenvolvida em diferentes aspectos do cotidiano, em relação aos parâmetros: sócio-ambientais (moradia, transporte, segurança, assistência médica, trabalho e remuneração, educação, lazer, meio ambiente saudável) e individuais (hereditariedade, hábitos alimentares, controle de estresse, atividade física e comportamento preventivo). Para que um indivíduo tenha uma boa qualidade de vida é necessário que ele tenha saúde, que segundo a Organização Mundial de Saúde (OMS), significa bem estar físico, social e psíquico.

Silveira Júnior (2001) afirma que a modalidade mais adequada para diminuir a incapacidade muscular e suas consequências na $3^{\circ}$ terceira idade é a musculação, pois aumenta ou melhora os níveis de força, preservam os tecidos musculares e previnem e auxiliam o tratamento da osteoporose. Ressalta ainda que, a intenção não é transformar o idoso em atleta, mas fazer o suficiente para que ele consiga subir uma escada, levantar de uma cadeira, caminhar com segurança, realizar as tarefas de seu cotidiano, que se tornam mais difíceis de serem realizadas devidas principalmente à perda de força e equilíbrio. Dessa forma ele se torna mais auto-confiante melhorando dessa forma sua vida de uma forma geral.

Segundo Leite (2000), a atividade física regular torna o idoso mais dinâmico e com menor incidência de doenças. Com isso o indivíduo da terceira idade terá melhor qualidade de vida e autoestima. Uma das atividades mais recomendadas para o idoso é a musculação, a qual mantém e até mesmo pode aumentar a força muscular, melhorando os movimentos básicos diários.

Diante disso, a atividade física parece ser sem dúvida, um dos meios para se evitar que o homem passe por um processo normal de envelhecimento para um envelhecimento mais saudável. Através desta pratica, muito dos problemas dos idosos podem ser amenizados, proporcionando-lhes uma vida saudável e tranquila.

Em uma pesquisa realizada por Matsudo et al., (2004), as pessoas do sexo feminino com mais de 50 anos de idade realizavam atividade física por: indicação médica $(38,3 \%)$, amigos $(33,3 \%)$, familiares $(10,4 \%)$, procura por companhia $(10,4 \%)$. Pode-se perceber através destes dados a preocupação do idoso com sua saúde ou recuperação em primeiro lugar. Os autores assinalam a importância que os idosos atribuem a sua saúde, preocupados em buscar uma vida com mais qualidade. O "fazer amigos" também é um dado importante, que é abordado por Geis (2003), 
quando idoso se torna participante de uma atividade física ele não busca somente a saúde, mas também a socialização. A atividade física pode ser um meio contra o isolamento social e a solidão, auxiliando contra o tédio, podendo compensar a redução das relações sociais e oferecer a substituição do "status" ora determinado pela atividade e posição profissional.

A capacidade de interagir socialmente é fundamental para o idoso, para que ele possa conquistar e manter as redes de apoio social e garantir maior qualidade de vida. As influências sociais da família e amigos são de extrema importância à manutenção da atividade física, pois esse suporte social incentiva o praticante a manter o interesse em continuar fisicamente ativo.

O envelhecimento é um processo natural, onde o organismo perde suas funções, e apresenta alterações como cabelos grisalhos, pele flácida e com manchas, perda de massa muscular e peso. Entretanto, muitos idosos querem mostrar que sua idade não influencia a sua auto-estima e sua imagem social. Neste sentido é importante separarmos o conceito de idade cronológica e psicológica, definido-as na forma em que o individuo interage com o meio e aceita seu processo de envelhecimento. Assim também pode ser avaliada a idade cronológica (números de anos transcorridos desde o nascimento) e social de acordo com o modo em que o indivíduo consegue ter sua adaptação na sociedade (PAPALÉO NETTO; CARVALHO FILHO; SALLES, 2006).

Gáspari e Schwartz (2005) ressaltam que fatores subjetivos como valores, atitudes e emoções são considerados importantes no enredo social, enfatizando que vivências no lazer possibilitam uma oportunidade para que as relações sociais aumentem. Esses autores realizaram um estudo com 20 idosos participantes do Programa Ativa "Idade": uma proposta de ação educativa, no âmbito do lazer, da cidade de Araras - São Paulo, tendo como objetivo principal identificar aspectos emocionais na percepção de idosos durante vivências do lazer. As respostas confirmam a hipótese de que experiências no lazer são capazes de contemplar a gama de necessidades e expectativas do homem no aspecto emocional e psicológico, contribuindo assim, no contexto social.

\section{Aspectos subjetivos do envelhecimento}

Atualmente o envelhecimento abrange um amplo campo de pesquisas e estudos, pois este possui uma dimensão existencial que se reveste de características biopsíquicas e socioculturais, por isso, sua análise deve ser realizada com base na dimensão biológica, sociológica e subjetiva (SHEPARD, 2003).

Mudanças no comportamento sociocultural já é presente, ou seja, o Brasil, um pais jovem que conta com $8 \%$ de sua população tendo mais de 60 anos, segundo epidemiologistas, em meados do ano 2025, ocupará a sexta posição mundial em número de idosos e a primeira posição da América Latina. Para isso, os idosos necessitam de cuidados e uma boa qualidade de vida, tornando-a prazerosa, digna e confortável.

Sintomas depressivos podem aparecer em decorrência de diversas patologias, em vigência do uso de vários medicamentos, ou após o início de outras doenças psiquiátricas como: transtorno obsessivo compulsivo ou síndrome do pânico. A qualidade de vida é considerada como a percepção do indivíduo de sua posição na vida, no contexto da cultura e sistema de valores nos quais vive e em relação aos seus objetivos, expectativas, padrões e preocupações.

Atualmente pessoas com mais de 60 anos estão exercendo papel ativo na sociedade e desenvolvem atividades que não eram comuns, até pouco tempo atrás, devido ao preconceito, estigmas e visão de incapacidade destes sujeitos pela sociedade, no qual inclui também a prática de atividades físicas (SILVA JÚNIOR; VELARDI, 2009).

Evidências científicas comprovam a importância da atividade física como um recurso fundamental no processo preventivo de doenças, melhora dos níveis de aptidão física, diminuição nos percentuais de gordura corporal, manutenção das capacidades funcionais. Além disso, a prática da atividade física regular tem sido descrita na literatura como um excelente meio de atenuar a degeneração provocada pelo processo de envelhecimento nos domínios físicos, psicológicos e sociais (CAPODAGLIO et al., 2006; MATSUDO et al., 2004). 
Em relação aos aspectos subjetivos, Matsudo et al. (2004) e Okuma (2002) afirmaram que a prática de atividade física é de fundamental importância neste estágio da vida, e, através dela, o idoso poderá obter benefícios tais como: melhora do auto-conceito; melhora da auto-estima; melhora da imagem corporal; contribui no desenvolvimento da auto-eficácia; diminuição do estresse, ansiedade; melhora da tensão muscular, insônia; diminuição do consumo de medicamentos; melhora das funções cognitivas e da socialização.

De acordo com o Posicionamento Oficial de Exercício e Atividade Física para Pessoas Idosas do American College Of Sports Medicine (ACSM) a prática da atividade física regular é considerada uma ferramenta indispensável nos aspectos psicológicos do envelhecimento, principalmente em relação à depressão, ansiedade e estresse que são consideradas questões de saúde pública.

Segundo Okuma (1998), a atividade física é considerada como um importante recurso para os idosos lidarem com situações de estresse. A autora aborda dois pontos, um referente ao maior bem estar físico resultante da atividade física, o que os levou a se sentirem mais fortalecidos e dispostos para enfrentar difíceis eventos da vida, o outro se refere à atividade física como atividade desafiadoras.

A auto-estima é entendida como positiva e negativa, a positiva, segundo Branden (2001), é aquela que existe a partir da valorização pessoal, de atitudes positivas em relação a viver e ser feliz, para que se possa desfrutar da vida. A negativa indica que o indivíduo sente medo de assumir riscos, do fracasso, sente-se incapaz e incompetente para realizar algo, vive se criticando, achando defeitos em si mesmo. Em relação ao círculo de amizades a auto-estima negativa leva a pessoa a se afastar do seu grupo social por sentir-se incapaz de manter um relacionamento profundo e significativo, levando-a ao isolamento.

Segundo Erbolato (2002), na velhice é normal que exista uma diminuição dos contatos sociais, provocando estado de solidão nos idosos. Porém, o engajamento de indivíduos desta faixa etária em programas de exercícios pode proporcionar a ampliação do círculo de amizades e, desta forma, contribuir no processo de integração social e elevando a auto-estima do idoso.

\section{Considerações Finais}

Por meio da análise bibliográfica foi possível perceber que a qualidade de vida dos idosos é influenciada por diversos fatores físicos, sociais e subjetivos, sendo o exercício físico, e mais especificamente a musculação, é grande responsável pela melhoria desses fatores fazendo com que eles acrescentem mais expectativas de uma vida melhor para cada idoso.

$\mathrm{Na}$ parte subjetiva a musculação é de grande importância já que através de melhorias fisiológicas como aumento muscular e melhor funcionamento das articulações o idoso é capaz de voltar a realizar atividade que antes não fazia. O fato mais importante para essa melhoria é a conquista da independência. A musculação, praticada em local socializador, pode auxiliar no relacionamento. Portanto, analisar a terceira idade de forma isolada é um equívoco, esse grupo tem que ser visto nessas três vertentes (físico, social e subjetivo), já que estão intrinsecamente interligados.

\section{Referências}

BRANDEN, Nathaniel. Auto-estima, como aprender a gostar de si mesmo. 39. ed. São Paulo: Saraiva 2001.

CAMPOS, M. A. Musculação Aplicada. Rio de Janeiro: Sprinter, 2000.

CAPODAGLIO, P. et al. Long-term strength training for communitydwelling people over 75: impact on muscle function, functional ability and life style. European Journal of Applied Physiology. v.100, n.5, p.535-542, 2006. 
ERBOLATO, R. M. P. L. Relações sociais na velhice. In: FREITAS, E. V. et al. (Org.). Tratado de geriatria e gerontologia. Rio de Janeiro: Guanabara-Koogan, 2002.

FRIED L. P. et al. Untangling the concepts of disability, frailty, and comorbidity implications for improved targeting and care. Journal of Gerontology: Medical Sciences. v.59, n.3, p.255-263, 2004.

GÁSPARI, J. C.; SCHWART, G. M. O idoso e a ressignificação emocional do lazer. Psicologia: teoria e pesquisa. v. 21, n. 1, p. 60-76, 2005.

GEIS, Pilar Pont. Atividade física e saúde na $3^{\text {a }}$ Idade. Porto Alegre: Artmed, 2003.

GUIMARÃES, M. N. G.; FARINATTI, P. T. V. Analise descritiva de variáveis teoricamente associadas ao risco de quedas em mulheres idosas. Revista Brasileira de Medicina do Esporte. v.11, n.5, p.299-305, 2005.

JETTE AM. Assesing disability in studies on physical activity. American Journal of Preventive Medicine. v.25, n.3 s.2 p.122-128, 2003.

LEITE, P. F. Aptidão física esporte e saúde. 3. ed. São Paulo: Robe, 2000.

LORDA, C. Raul; SANCHEZ, Carmem Delia. Recreação na $3^{\text {a }}$ idade. 3. ed. Rio de Janeiro: Sprint, 2001.

MATSUDO, S. M. Atividade Física e Envelhecimento: aspectos epidemiológicos. Revista Brasileira de Atividade Física e Saúde. v. 7, n. 1, p. 2-13, 2001.

Avaliação do idoso: física \& funcional. 2. ed. Londrina: Midiograf, 2005.

MATSUDO, S. M. et al. Estudo longitudinal-tracking de 4 anos - da aptidão física de mulheres da maioridade fisicamente ativas. Revista Brasileira de Ciência e Movimento. v.12, n.3, p.47-52, 2004.

MEIRELLES, Morgana E. Atividade física na 3a idade. 3. ed. Rio de Janeiro: Sprint, 2000.

MONTEIRO, Pedro Paulo. Envelhecer: histórias, encontros, transformações. Belo Horizonte: Autêntica, 2001.

NADEAU, M.; PÉRONNET, F. Fisiologia aplicada na atividade física. São Paulo: Manole, 1985.

NAHAS, V. M. Atividade, saúde e qualidade de vida: Conceitos e sugestões para um estilo de vida ativo. 2. ed. Londrina: Midiograf, 2001.

OKUMA, S. S. O idoso e a atividade física: fundamentos e pesquisa. Campinas: Papirus, 1998.

- Cuidados com o corpo: um modelo pedagógicode educação física para idosos. In:

FREITAS, E. V. et al. (Org.). Tratado de Geriatria e Gerontologia. Rio de Janeiro: GuanabaraKoogan. p. 1092-1100, 2002. 
PAPALÉO NETTO, M; CARVALHO FILHO, E. T; SALlES, R. F. N. Fisiologia do Envelhecimento. Geriatria - Fundamentos, Clínica e Terapêutica. 2. ed. São Paulo: Atheneu, 2006.

SHEPHARD, R. J. Envelhecimento, atividade física e saúde. São Paulo: Phorte, 2003.

SILVA JÚNIOR, A. P.; VELARDI, M. Adesão de idosos a programas de atividades físicas - a questão da promoção da saúde. The FIEP Bulletin, v.79, p.155-157, 2009.

SILVEIRA JÚNIOR, Antônio Augusto. Musculação aplicada ao envelhecimento. Curitiba: Instituto Lyon, 2001.

VALE, R. G. S.; NOVAES, J. S.; DANTAS, E. H. M. Efeitos do treinamento de força e de flexibilidade sobre a autonomia de mulheres senescentes. Revista Brasileira de Ciência e Movimento. v.13, n.2, p.33-40, 2005.

ZAGO, A. S.; GOBBI, S. Valores normativos de aptidão funcional de mulheres de 60 a 70 anos. Revista Brasileira de Ciência e Movimento. v.11, n.2, p.77-87, 2003. 